\title{
Adverse drug events in hospitalized patients in Brazil: Integrative literature review
}

\author{
Eventos adversos em pacientes hospitalizados no Brasil: Revisão integrativa da literatura \\ Eventos adversos por fármacos en pacientes hospitalizados en Brasil: Revisión integradora de la
}

literatura

Received: 03/12/2021 | Reviewed: 03/18/2021 | Accept: 03/21/2021 | Published: 03/29/2021

\author{
Chriscia Jamilly Pinto de Sousa \\ ORCID: https://orcid.org/0000-0002-6996-7717 \\ Federal University of Pará, Brazil \\ E-mail: jamillysousa@ufpa.br \\ Ana Cristina Lo Prete \\ ORCID: https://orcid.org/0000-0002-2874-5296 \\ São Judas Tadeu University, Brazil \\ E-mail: ana.loprete@saojudas.br \\ Amanda Gabriele Piedade Gomes \\ ORCID: https://orcid.org/0000-0002-6329-3176 \\ University of Amazon, Brazil \\ E-mail: amandagomes464@yahoo.com \\ Eline Fernandes Ribeiro de Castro \\ ORCID: https://orcid.org/0000-0002-4539-5646 \\ Federal University of Pará, Brazil \\ E-mail: fernandeseline@hotmail.com \\ Carolina Heitmann Mares Azevedo Ribeiro \\ ORCID: https://orcid.org/0000-0002-9457-2733 \\ Federal University of Pará, Brazil \\ E-mail: carolmheitmann@hotmail.com
}

\begin{abstract}
Objective: Analyzing studies related to Adverse Drug Events (ADE) in hospitalized patients in Brazil. Method: integrative review, for which the National Library of Medicine (PubMed), Biblioteca Virtual de Saúde (BVS), Repository of the Fundação Oswaldo Cruz (FIOCRUZ) and Capes Journals databases were selected for searching the studies primary, with the descriptors: Patient Safety, Drug-related side effects and adverse reactions, Patient harm, Hospitalization, Inpatients. Results: Twenty articles were identified, 50\% (10) were from the southeast region of Brazil. Regarding the methodology adopted to identify the ADEs, 70\% (14) used the retrospective data review. The trackers were used in $5(25 \%)$ articles, 3 of which used the triggers proposed by the Institute for Healthcare Improvement, and the other 2 the trackers used were proposed by the researchers themselves. The number of adverse drug reactions ranged from 12 to 96, while the occurrence of AE ranged from 4 to 122 . In one study $50 \%$ (41) of participants had at least one adverse drug event. Conclusion: There is great variability in scientific production in Brazil, and in the occurrence of Adverse Drug Events as well. There is a need for strategies to identify these events and create strategies to promote patient safety.
\end{abstract}

Keywords: Drug-related side effects and adverse reactions; Pharmacovigilance; Quality indicators, Health care.

\section{Resumo}

Objetivo: Analisar estudos relacionados a Eventos Adversos a Medicamento (EAM) em pacientes hospitalizados no Brasil. Método: revisão integrativa, para a qual foram selecionadas as bases de dados da Biblioteca Nacional de Medicina (PubMed), Biblioteca Virtual em Saúde (BVS), Repositório da Fundação Oswaldo Cruz (FIOCRUZ) e Periódicos Capes para a busca dos estudos primários, com os descritores: Patient Safety, Drug-related side effects and adverse reactions, Patient harm, Hospitalization, Inpatients. Resultados: Foram identificados 20 artigos, 50\% (10) da região sudeste do Brasil. Em relação à metodologia adotada para identificar os ADEs, 70\% (14) utilizaram a revisão retrospectiva dos dados. Os rastreadores foram utilizados em 5 (25\%) artigos, dos quais 3 utilizaram os gatilhos propostos pelo Institute for Healthcare Improvement e os outros 2 os rastreadores utilizados foram propostos pelos próprios pesquisadores. O número de reações adversas a medicamentos variou de 12 a 96 , enquanto a ocorrência de EA variou de 4 a 122. Em um estudo, 50\% (41) dos participantes tiveram pelo menos um evento adverso a medicamentos. Conclusão: Há grande variabilidade na produção científica no Brasil, bem como na ocorrência de Eventos Adversos a Medicamentos. São necessárias estratégias para identificar esses eventos e criar estratégias para promover a segurança do paciente. 
Palavras-chave: Efeitos colaterais e reações adversas relacionados a medicamentos; Farmacovigilância; Indicadores de qualidade em assistência à saúde.

\section{Resumen}

Objetivo: Analizar estudios relacionados con Eventos Adversos a Medicamentos (AME) en pacientes hospitalizados en Brasil. Método: revisión integradora, para lo cual se seleccionaron las bases de datos de la Biblioteca Nacional de Medicina (PubMed), Biblioteca Virtual en Salud (BVS), Repositorio de la Fundación Oswaldo Cruz (FIOCRUZ) y Revistas Capes para la búsqueda de estudios primarios, con los descriptores : Patient Safety, Drug-related side effects and adverse reactions, Patient harm, Hospitalization, Inpatients.. Resultados: Se identificaron veinte artículos, 50\% (10) de la región sureste de Brasil. En cuanto a la metodología adoptada para identificar las EAM, el 70\% (14) utilizó la revisión retrospectiva de los datos. Los rastreadores se utilizaron en 5 (25\%) artículos, de los cuales 3 utilizaron los activadores propuestos por el Instituto para la Mejora de la Salud y los otros 2 seguidores utilizados fueron propuestos por los propios investigadores. El número de reacciones adversas a medicamentos varió de 12 a 96, mientras que la aparición de EA varió de 4 a 122. En un estudio, el 50\% (41) de los participantes tuvo al menos un evento adverso de medicamentos. Conclusión: Existe una gran variabilidad en la producción científica en Brasil, así como en la ocurrencia de Eventos Adversos a los Medicamentos. Se necesitan estrategias para identificar estos eventos y crear estrategias para promover la seguridad del paciente.

Palabras clave: Efectos colaterales y reacciones adversas relacionados con medicamentos; Farmacovigilancia; Indicadores de calidad de la atención de salud.

\section{Introduction}

Patient safety, according to the World Health Organization (WHO), is one of the quality attributes of health services. According to WHO (2009), it aims to "reduce the risk of unnecessary harm associated with healthcare to the minimum acceptable".

According to the Institute of Medicine (IOM) (2000) and the National Health Surveillance Agency (2013), the term "patient safety" started to be discussed internationally with the launch of the report "To Err is Human" prepared by the Institute of Medicine (IOM), which found, through a retrospective analysis of medical records of patients admitted to hospitals in New York, Utah and Colorado, that about 100,000 people die each year in the United States due to ADEs.

WHO (2002) and Mendes, Martins, Rozenfeld and Travassos (2009), state that Adverse Events (AE) can be defined as any incident that results in unintentional damage resulting from care, unrelated to the patient's natural course of illness. Thus, Adverse Drug Events (AME) are among the three most common causes of AS. AEs include Adverse Drug Reactions (ADRs), defined as any harmful or unwanted effects after drug administration in doses commonly used by humans for the prophylaxis, diagnosis or treatment of a disease.

Vicent (2010) ensures that in the United States, at least one medication error occurs each day in each patient. These errors, although almost predictable, can trigger events. Thus, hospitalized patients are particularly susceptible to the occurrence of AMI.

The study by Kimelblatt et al. (1988) indicated the occurrence of ADEs in 10 to $20 \%$ of all hospitalized patients. Classen found that $2.3 \%$ of hospitalized patients had their clinical condition aggravated by ADEs and 3.5\% of these led to death. In these studies, it is possible to identify the increase in hospital stay and, consequently, the cost of treatment by $174 \%$. Also concluded that $50 \%$ of expenditures could be avoided by DE prevention measures (Classen, Pestotnick, Evans, Lloyd \& Burke, 1997). The studies by Venulet \& Ham (1996), Nunes (2000) and Brasil (2013) indicate that in Brazil, between 2006 and 2013, the report of the Health Surveillance Notification System (NOTIVISA) showed a total of 103,887 EA, 38,730 were ADEs and of these, $14.8 \%$ to $59 \%$ could be avoided.

According to WHO (2002), among the methods of identifying AMI, are voluntary notification (passive search) and intensive monitoring (active search).

As explained by Dolores-Menendez et al. (2019), Spontaneous Notification is a low cost method, in which professionals and / or patients spontaneously report the occurrence of AE. The main limitation of this methodology is 
underreporting.

In the active search, $\mathrm{AE}$ is identified through a retrospective and / or prospective review of the patient's medical record based on criteria previously defined by the institution. This method is more effective in identifying AE, but it requires time, human and financial resources to be well employed. In addition, the effectiveness of the method, when performed retrospectively, depends on the quality of the professionals' records, which also becomes a limiting factor (WHO, 2002; Griffin \& Resar, 2009; Classen et al, 2011).

Therefore, identifying and knowing the occurrence of ADRs and ADEs are requirements to make health services more secure and financially healthy. Therefore, this review aims to analyze studies related to Adverse Drug Events (AME) in hospitalized patients in Brazil.

\section{Methods}

Also based on studies by Haukland, Plessen, Nieder and Vonen (2017). This work is an integrative review based on the research question: "How is the identification of ADRs made and what is the profile of AS in hospitalized patients in Brazil?". This was delimited by the PICO strategy (acronym for Patient, Intervention, Comparison, Results). The steps taken in preparing the study were: elaboration of the research question, sampling or search in the literature of primary studies, data extraction, evaluation of primary studies, interpretation of results, presentation of the review.

The primary studies were searched from July to August 2019, in the following databases: National Library of Medicine of the National Institutes of Health (PubMed), Virtual Health Library (VLH / BVS), Repositório da Fundação Oswaldo Cruz (FIOCRUZ) and Capes Journals.

The controlled descriptors selected in the Health Sciences Descriptors (HSD) of the Virtual Health Library (VLH / BVS) and HSD in PubMed were "Patient Safety, Side Effects and Adverse Reactions Related to Medicines, Patient Damage, Hospitalization, Inpatients " (in English). The descriptors were combined using the Boolean operators, which obtained the following searches: "patient safety AND side effects and adverse reactions related to drugs and damage to the patient and hospital and inpatients", "patient safety AND related side effects and adverse reactions to medicines ". OR damage to the patient AND Hospitalization OR Inpatients".

The inclusion criteria were articles whose main and / or secondary objective was to identify ADRs and / or ADEs in hospitalized patients. Regarding the period, we selected the articles published in the last five years (2014 to 2019). The search was conducted in Portuguese, Spanish and English. The exclusion criteria were: studies carried out in emergency rooms and outpatient services in hospitals, case reports and studies that did not differentiate the types of adverse events.

The choice of articles was divided into three stages:

1) Search for articles using descriptors in the databases, filtering the period of 5 years.

2) Reading of titles and abstracts by two independent reviewers who decided to include or exclude the article. In case of disagreement, a third reviewer was invited.

3) Reading of the articles in full by the reviewers, in order to identify whether the article answered the proposed question.

Were identified 194 articles in the four databases. After applying the criteria, 174 articles were excluded, for the following reasons: Duplicates (17), Secondary Studies and / or Case Report (8), Other Countries (6), Does not Answer the Proposed Question (116) and Studies in Outpatients (27). Thus, 20 articles were qualified for this review.

\section{Results}

The information extracted from the primary studies included in the review is shown in Table 1. 
Table 1 - Information extracted from the primary studies.

\begin{tabular}{|c|c|c|c|c|c|c|c|c|c|c|c|c|c|}
\hline $\mathbf{N}^{\circ}$ & Title & $\begin{array}{c}\text { Autor } \\
\text { Ano }\end{array}$ & $\begin{array}{l}\text { Language } \\
\text { Place }\end{array}$ & $\begin{array}{c}\text { Population } \\
\text { Sample }\end{array}$ & Objective & Method & Gender & $\begin{array}{l}\text { Length } \\
\text { of stay }\end{array}$ & Age & $\begin{array}{l}\mathbf{N}^{\circ} \text { of } \\
\text { ADE }\end{array}$ & $\mathrm{N}^{0}$ of ADR & Main Drugs & Risk factors \\
\hline 1 & $\begin{array}{c}\text { Factors associated } \\
\text { with drug interactions } \\
\text { in elderly hospitalized } \\
\text { in high complexity } \\
\text { hospital. }\end{array}$ & $\begin{array}{c}\text { Veloso, } \\
\text { Figueiredo, } \\
\text { Barroso, } \\
\text { Nascimento \& } \\
\text { Reis (2019) }\end{array}$ & $\begin{array}{l}\text { Portuguese } \\
\text { Minas } \\
\text { Gerais }\end{array}$ & 237 seniors & $\begin{array}{c}\text { Determine the } \\
\text { frequency of possible } \\
\text { drug interactions } \\
\text { (PID) in hospitalized } \\
\text { elderly and associated } \\
\text { factors. }\end{array}$ & $\begin{array}{l}\text { Retrospective } \\
\text { analysis of } \\
\text { medical records. } \\
\text { Period: } 1 \text { year }\end{array}$ & $\begin{array}{c}120 \\
(50,6 \%) \\
\text { male }\end{array}$ & $\begin{array}{c}\leq 12 \\
\text { days, } \\
(51,5 \%)\end{array}$ & 70 years & - & $\begin{array}{l}51 \text { ADR } \\
16(6,8 \%) \\
\text { related to drug } \\
\text { interaction }\end{array}$ & - & - \\
\hline 2 & $\begin{array}{l}\text { Incident analysis } \\
\text { occurrence related to } \\
\text { potentially dangerous } \\
\text { medicines distributed } \\
\text { in teaching hospital. }\end{array}$ & $\begin{array}{l}\text { Basile, et al. } \\
\text { (2019) }\end{array}$ & $\begin{array}{l}\text { Portuguese } \\
\text { São Paulo }\end{array}$ & $\begin{array}{c}786 \\
\text { Notifications }\end{array}$ & $\begin{array}{c}\text { Analyze the reports } \\
\text { of incidents related to } \\
\text { potentially dangerous } \\
\text { drugs dispensed in a } \\
\text { teaching hospital in } \\
\text { the interior of São } \\
\text { Paulo. }\end{array}$ & $\begin{array}{l}\text { Retrospective } \\
\text { analysis of } \\
\text { notifications. } \\
\text { Period: } 5 \text { years }\end{array}$ & $\begin{array}{c}226 \\
(28,72 \%) \\
\text { male and } \\
226 \\
(28,72 \%) \\
\text { sexo } \\
\text { female }\end{array}$ & - & - & - & $\begin{array}{c}30 \\
\text { ADR15,95\% } \\
\text { of notifications }\end{array}$ & $\begin{array}{l}\text { Drugs That } \\
\text { Act On: } \\
\text { Nervous } \\
\text { System } 67 \\
(35,63 \%)\end{array}$ & - \\
\hline 3 & $\begin{array}{l}\text { Increase of } 10 \% \text { in the } \\
\text { Rate of Adverse Drug } \\
\text { Reactions for Each } \\
\text { Drug Administered in } \\
\text { Hospitalized Patients. }\end{array}$ & $\begin{array}{c}\text { Ribeiro, Motta, } \\
\text { Marcondes- } \\
\text { Fonseca, Kalil- } \\
\text { Filho \& } \\
\text { Giavina- } \\
\text { Bianchi (2018) }\end{array}$ & $\begin{array}{l}\text { English } \\
\text { São Paulo }\end{array}$ & 472 pacients & $\begin{array}{l}\text { Assess risk factors, } \\
\text { incidence and } \\
\text { severity of adverse } \\
\text { drug reactions in } \\
\text { inpatients }\end{array}$ & $\begin{array}{c}\text { Prospective } \\
\text { analysis of } \\
\text { medical records, } \\
\text { patients } \\
\text { hospitalized for } \\
\text { more than } 48 \\
\text { hours. } \\
\text { Duration: } 3 \text { years } \\
\text { Causality: } \\
\text { Naranjo } \\
\text { Algorithm }\end{array}$ & $\begin{array}{c}239 \\
(51,5 \%) \\
\text { female. }\end{array}$ & $\begin{array}{l}16,8 \\
\text { days }\end{array}$ & $\begin{array}{c}57,5 \\
\text { years }( \pm \\
19,9)\end{array}$ & - & $\begin{array}{l}94 \mathrm{ADR} \text { in } 75 \\
\text { pacients }(16 \%)\end{array}$ & $\begin{array}{c}\text { Antibiotics } \\
(21.2 \%) \\
\text { Opioids } \\
(13.8 \%) \\
\text { Iodinated } \\
\text { contrast media } \\
(10.6 \%)\end{array}$ & $\begin{array}{l}\text { Chronic renal failure; } \\
\text { Prolonged length of stay; } \\
\text { Higher number of } \\
\text { medications on } \\
\text { admission; } \\
\text { For each drug introduced } \\
\text { during hospitalization, } \\
\text { there was a } 10 \% \text { increase } \\
\text { in the adverse drug } \\
\text { reaction rate. }\end{array}$ \\
\hline 4 & $\begin{array}{l}\text { Hospitalizations and } \\
\text { deaths from drug } \\
\text { poisoning and adverse } \\
\text { reactions in Brazil: an } \\
\text { analysis from } 2000 \text { to } \\
2014 .\end{array}$ & $\begin{array}{c}\text { Santos \& } \\
\text { Boing (2018) }\end{array}$ & $\begin{array}{l}\text { Portuguese } \\
\text { Brazil }\end{array}$ & - & $\begin{array}{c}\text { Describe the } \\
\text { tendency of mortality } \\
\text { and hospitalizations } \\
\text { for these diseases in } \\
\text { Brazil, between } 2000 \\
\text { and } 2014 .\end{array}$ & $\begin{array}{c}\text { Retrospective } \\
\text { analysis of data } \\
\text { from MIS } \\
\text { (Mortality } \\
\text { Information } \\
\text { System) and } \\
\text { SIH-SUS (SUS } \\
\text { Hospital } \\
\text { Information } \\
\text { System) CID-10 } \\
\text { Period: } 4 \text { years }\end{array}$ & - & - & - & $\begin{array}{c}0,4 \% \\
\text { hospital } \\
\text { admission } \\
\text { s } \\
\text { proportion } \\
\text { al to } \\
100,000 \\
\text { inhabitants }\end{array}$ & - & - & - \\
\hline
\end{tabular}


Research, Society and Development, v. 10, n. 4, e3410413818, 2021

(CC BY 4.0) | ISSN 2525-3409 | DOI: http://dx.doi.org/10.33448/rsd-v10i4.13818

\begin{tabular}{|c|c|c|c|c|c|c|c|c|c|c|c|c|c|}
\hline 5 & $\begin{array}{l}\text { Preventable adverse } \\
\text { drug events in } \\
\text { critically ill HIV } \\
\text { patients: Is the } \\
\text { detection of potential } \\
\text { drug-drug interactions } \\
\text { a useful tool? }\end{array}$ & $\begin{array}{c}\text { Ramos, } \\
\text { Japiassu, } \\
\text { Bozza \& } \\
\text { Guaraldo } \\
\text { (2018) }\end{array}$ & $\begin{array}{l}\text { English } \\
\text { Rio de } \\
\text { Janeiro }\end{array}$ & $\begin{array}{l}62 \text { patients } \\
\text { with HIV / } \\
\text { AIDS in the } \\
\text { ICU }\end{array}$ & $\begin{array}{l}\text { Develop a strategy to } \\
\text { identify AE } \\
\text { associated with drug } \\
\text { interactions by } \\
\text { analyzing } \\
\text { prescriptions of } \\
\text { critically ill patients }\end{array}$ & $\begin{array}{l}\text { Retrospective } \\
\text { analysis of } \\
\text { medical records. } \\
\text { Causality: } \\
\text { Naranjo } \\
\text { Algorithm } \\
\text { Period: } 2 \text { years }\end{array}$ & 72,6 male & 13 days & $\begin{array}{c}37,5 \\
\text { years }\end{array}$ & $\begin{array}{l}9 \% \text { of } \\
\text { interaction } \\
\text { s resulted } \\
\text { in } 24 \mathrm{MI} \\
\text { in } 16 \\
(25.8 \%) \\
\text { patients }\end{array}$ & $\begin{array}{l}20 \mathrm{ADR} \\
(83,3 \%)\end{array}$ & Anticonvulsant & - \\
\hline 6 & $\begin{array}{l}\text { Risk factors associated } \\
\text { with hypoglycemia } \\
\text { and analysis of adverse } \\
\text { events in intensive } \\
\text { therapy. }\end{array}$ & $\begin{array}{l}\text { Roque, Silva, } \\
\text { Santos \& Melo } \\
\text { (2018) }\end{array}$ & $\begin{array}{l}\text { Portuguese } \\
\text { Rio de } \\
\text { Janeiro }\end{array}$ & $\begin{array}{l}355 \text { adults in } \\
\text { ICU }\end{array}$ & $\begin{array}{l}\text { Evaluate the } \\
\text { predictive factors } \\
\text { associated with the } \\
\text { occurrence of severe } \\
\text { hypoglycemia and to } \\
\text { analyze adverse } \\
\text { events related to } \\
\text { insulin use and oral } \\
\text { hypoglycemic agents } \\
\text { in ICU patients. }\end{array}$ & $\begin{array}{c}\text { Prospective } \\
\text { analysis of } \\
\text { medical records } \\
\text { of ICU patients. } \\
\text { Period: 1 year } \\
\text { IHI adaptation } \\
\text { Causality: } \\
\text { Naranjo } \\
\text { Algorithm }\end{array}$ & $\begin{array}{c}189(53 \%) \\
\text { Female }\end{array}$ & $\begin{array}{c}\text { Enferma } \\
\text { ria: } 9,83 \\
\text { days } \\
\\
\text { UTI } \\
17,5 \\
\text { days }\end{array}$ & $\begin{array}{l}60,18 \\
\text { years }\end{array}$ & $\begin{array}{l}120 \text { events } \\
\text { of severe } \\
\text { hypoglyce } \\
\text { mia }\end{array}$ & - & - & $\begin{array}{l}\text { Presence of liver disease; } \\
\text { Sepsis; } \\
\text { Occurrence of adverse } \\
\text { events; }\end{array}$ \\
\hline 7 & $\begin{array}{l}\text { Adverse events to } \\
\text { drugs identified in } \\
\text { hospitalized patients in } \\
\text { Brazil according to } \\
\text { codes of the } \\
\text { International } \\
\text { Classification of } \\
\text { Diseases (ICD-10). }\end{array}$ & $\begin{array}{l}\text { Martins, } \\
\text { Giordani, } \\
\text { Guaraldo, } \\
\text { Tognoni \& } \\
\text { Rozenfeld } \\
\text { (2018) }\end{array}$ & $\begin{array}{c}\text { English } \\
2018 \\
\text { Brazil }\end{array}$ & $\begin{array}{l}55.604 .537 \\
\text { hospital } \\
\text { admission } \\
\text { records }\end{array}$ & $\begin{array}{l}\text { It examined the } \\
\text { potential of applying } \\
\text { CID-10 codes }\left(10^{\mathrm{th}}\right. \\
\text { revision of the } \\
\text { International } \\
\text { Classification of } \\
\text { Diseases) in a } \\
\text { national public health } \\
\text { system database } \\
\text { (SIH-SUS).I }\end{array}$ & $\begin{array}{l}\text { Retrospective } \\
\text { analysis of data } \\
\text { from SIM } \\
\text { (Mortality } \\
\text { Information } \\
\text { System) and } \\
\text { SIH-SUS (SUS } \\
\text { Hospital } \\
\text { Information } \\
\text { System) } \\
\text { CID Code } \\
\text { Period: } 4 \text { years }\end{array}$ & - & - & - & $\begin{array}{c}273,440 \\
(0.49 \%) \\
\text { were } \\
\text { related to } \\
\text { at least } \\
\text { one ADE }\end{array}$ & - & - & - \\
\hline 8 & $\begin{array}{c}\text { Triggers for active } \\
\text { surveillance of adverse } \\
\text { drug } \\
\text { events in newborns. }\end{array}$ & $\begin{array}{c}\text { Fabretti, } \\
\text { Brassica, } \\
\text { Cianciarullo \& } \\
\text { Romano- } \\
\text { Lieber (2018) }\end{array}$ & $\begin{array}{c}\text { Portuguese } \\
\text { São Paulo }\end{array}$ & $\begin{array}{l}125 \\
\text { Neonates in } \\
\text { the Neonatal } \\
\text { Intensive } \\
\text { Care Unit } \\
\text { (ICU) and } \\
\text { Conventiona } \\
1 \text { Neonatal } \\
\text { Intermediate } \\
\text { Care Unit } \\
\text { (UCINCo). }\end{array}$ & $\begin{array}{l}\text { Verify the application } \\
\text { and performance of } \\
\text { triggers for adverse } \\
\text { drug events in } \\
\text { hospitalized } \\
\text { newborns. }\end{array}$ & $\begin{array}{c}\text { Prospective } \\
\text { analysis of } \\
\text { medical records } \\
\text { of patients } \\
\text { admitted to the } \\
\text { ICU and neonatal } \\
\text { ICU. } \\
\text { Period: } 7 \text { months } \\
\text { Own Trackers }\end{array}$ & - & - & - & $\begin{array}{c}115 \\
\mathrm{ADE}\end{array}$ & - & - & - \\
\hline
\end{tabular}


Research, Society and Development, v. 10, n. 4, e3410413818, 2021

(CC BY 4.0) | ISSN 2525-3409 | DOI: http://dx.doi.org/10.33448/rsd-v10i4.13818

\begin{tabular}{|c|c|c|c|c|c|c|c|c|c|c|c|c|c|}
\hline 9 & $\begin{array}{l}\text { Risk factors for death } \\
\text { in patients with non- } \\
\text { infectious adverse } \\
\text { events. }\end{array}$ & $\begin{array}{c}\text { Gadelha, } \\
\text { Paixão, Prado, } \\
\text { Viana \& } \\
\text { Amaral (2018) }\end{array}$ & $\begin{array}{l}\text { English } \\
\text { Acre }\end{array}$ & $\begin{array}{l}792 \mathrm{ICU} \\
\text { adult } \\
\text { patients }\end{array}$ & $\begin{array}{c}\text { Identify risk factors } \\
\text { for death in patients } \\
\text { who have } \\
\text { experienced non- } \\
\text { infectious adverse } \\
\text { events. }\end{array}$ & $\begin{array}{c}\text { Retrospective } \\
\text { analysis of } \\
\text { medical records; } \\
\text { Period: } 1 \text { year } \\
\text { and } 11 \text { months }\end{array}$ & $\begin{array}{c}466 \\
(58,8 \%) \\
\text { male }\end{array}$ & $\begin{array}{c}458 \\
(58,3) \\
\leq 7 \\
\text { days }\end{array}$ & $\begin{array}{l}>50 \\
\text { years }\end{array}$ & $\begin{array}{c}\text { From the } \\
\text { AE } \\
\text { identified } \\
94 \\
(17.7 \%) \\
\text { Medicatio } \\
\mathrm{n} \text { and } \\
\text { blood } \\
\text { transfusio } \\
\mathrm{n}\end{array}$ & - & $\begin{array}{c}40(43.9 \%) \\
\text { Antimicrobials } \\
16(17.6 \%) \\
\text { Anti or } \\
\text { procoagulants } \\
\\
07(7.7 \%) \\
\text { Sedation / } \\
\text { Analgesia } \\
\\
05(5.5 \%) \\
\text { Electrolytes }\end{array}$ & - \\
\hline 10 & $\begin{array}{l}\text { Factors associated } \\
\text { with adverse drug } \\
\text { reactions in older } \\
\text { inpatients in teaching } \\
\text { hospital. }\end{array}$ & $\begin{array}{l}\text { Figueiredo, } \\
\text { Groia, Barroso, } \\
\text { Nascimento \& } \\
\text { Reis (2017) }\end{array}$ & $\begin{array}{l}\text { English } \\
\text { Minas } \\
\text { Gerais }\end{array}$ & $\begin{array}{l}237 \text { elderly } \\
\text { hospitalized } \\
\text { for more } \\
\text { than five } \\
\text { days. }\end{array}$ & $\begin{array}{l}\text { Determine the } \\
\text { prevalence of ADRs } \\
\text { among elderly } \\
\text { patients and the } \\
\text { factors associated } \\
\text { with their occurrence. } \\
\text { Brazilian hospital } \\
\text { environment. }\end{array}$ & $\begin{array}{l}\text { Retrospective } \\
\text { analysis of } \\
\text { medical records; } \\
\text { Own trackers } \\
\text { Period: } 1 \text { year. }\end{array}$ & $\begin{array}{c}120 \\
(50,6 \%) \\
\text { male }\end{array}$ & 12 days & 70 years & - & $\begin{array}{l}62 \mathrm{ADR} \text { in } 50 \\
(21.1 \%) \text { of the } \\
\text { elderly }\end{array}$ & $\begin{array}{l}\text { Vancomycin ( } \mathrm{n} \\
=5), \text { Morphine } \\
(\mathrm{n}=4), \\
\text { Captopril }(\mathrm{n}= \\
\text { 4), Furosemide } \\
(\mathrm{n}=4) .\end{array}$ & $\begin{array}{l}\text { Cardiac insufficiency } \\
\text { Length of stay }>12 \text { days. }\end{array}$ \\
\hline 11 & $\begin{array}{c}\text { Risk factors for } \\
\text { adverse drug reactions } \\
\text { in pediatric inpatients: } \\
\text { A cohort study. }\end{array}$ & $\begin{array}{l}\text { Andrande, } \\
\text { Lobo \& Silva } \\
\text { (2017) }\end{array}$ & $\begin{array}{l}\text { English } \\
\text { Sergipe }\end{array}$ & $\begin{array}{l}173 \text { pediatric } \\
\text { patients } \\
\text { hospitalized } \\
\text { for more } \\
\text { than } 48 \\
\text { hours. }\end{array}$ & $\begin{array}{l}\text { Identify risk factors } \\
\text { for adverse drug } \\
\text { reactions (ADRs) in } \\
\text { pediatric patients. }\end{array}$ & $\begin{array}{l}\text { Prospective } \\
\text { analysis of } \\
\text { medical records, } \\
\text { team and patient } \\
\text { reports, and } \\
\text { spontaneous } \\
\text { reports; } \\
\text { Naranjo's } \\
\text { algorithm } \\
\text { Period: } 10 \\
\text { months }\end{array}$ & $\begin{array}{c}95 \\
(54,9 \%) \\
\text { male }\end{array}$ & 8 days & $\begin{array}{c}4,8 \\
\text { years }\end{array}$ & - & $\begin{array}{l}66 \mathrm{ADR} \text { in } 38 \\
(22 \%) \text { patients. }\end{array}$ & $\begin{array}{l}\text { Antiepileptic } \\
\quad \text { drugs; } \\
\text { Use of } \\
\text { systemic } \\
\text { antibiotics; } \\
\text { Use of } \\
\text { meglumine } \\
\text { antimoniate; }\end{array}$ & Previous history of ADR; \\
\hline 12 & $\begin{array}{c}\text { Epidemiological, } \\
\text { clinical and } \\
\text { evolutionary aspects of } \\
\text { tuberculosis among } \\
\text { elderly patients of a } \\
\text { university hospital in } \\
\text { Belém, Pará }\end{array}$ & Chaves (2017) & $\begin{array}{l}\text { Portuguese } \\
\text { Pará }\end{array}$ & $\begin{array}{l}82 \text { elderly } \\
\text { with } \\
\text { tuberculosis }\end{array}$ & $\begin{array}{c}\text { Evaluate the } \\
\text { epidemiological, } \\
\text { clinical and } \\
\text { evolutionary aspects } \\
\text { of tuberculosis in the } \\
\text { elderly in a } \\
\text { University Hospital } \\
\text { in the city of Belém, } \\
\text { Pará. }\end{array}$ & $\begin{array}{l}\text { Retrospective } \\
\text { analysis of } \\
\text { medical records. } \\
\text { Period: } 4 \text { years }\end{array}$ & $\begin{array}{c}\text { Male } \\
\text { predomina } \\
\text { nce }\end{array}$ & - & $\begin{array}{l}69,8 \\
\text { years }\end{array}$ & - & $\begin{array}{l}77 \text { ADR in } 41 \\
\text { (50\%) of the } \\
\text { elderly for TB }\end{array}$ & - & - \\
\hline
\end{tabular}


Research, Society and Development, v. 10, n. 4, e3410413818, 2021

(CC BY 4.0) | ISSN 2525-3409 | DOI: http://dx.doi.org/10.33448/rsd-v10i4.13818

\begin{tabular}{|c|c|c|c|c|c|c|c|c|c|c|c|c|c|}
\hline 13 & $\begin{array}{l}\text { Antimicrobial drug- } \\
\text { related problems in a } \\
\text { neonatal intensive } \\
\text { care unit }\end{array}$ & $\begin{array}{c}\text { Nunes, Xavier } \\
\& \text { Martins } \\
\text { (2017) }\end{array}$ & $\begin{array}{l}\text { Portuguese } \\
2017 \\
\text { Rio Grande } \\
\text { do Norte }\end{array}$ & $\begin{array}{l}85 \text { neonates } \\
\text { in the ICU, } \\
\text { hospitalized } \\
\text { for more } \\
\text { than } 24 \\
\text { hours. }\end{array}$ & $\begin{array}{l}\text { Determine the main } \\
\text { drug-related problems } \\
\text { in neonates using } \\
\text { antimicrobials. }\end{array}$ & $\begin{array}{c}\text { Prospective } \\
\text { analysis of } \\
\text { medical records. }\end{array}$ & $\begin{array}{c}58,5 \% \\
\text { male }\end{array}$ & - & - & & $\begin{array}{c}12 \text { RAM } \\
\text { represents } \\
15.2 \% \text { of } \\
\text { identified drug } \\
\text { related } \\
\text { problems }\end{array}$ & $\begin{array}{l}\text { Gentamicin } \\
\text { Amikacin } \\
\text { Cefazolin }\end{array}$ & - \\
\hline 14 & $\begin{array}{c}\text { Potentially } \\
\text { inappropriate } \\
\text { prescribing and the } \\
\text { risk of adverse drug } \\
\text { reactions in critically } \\
\text { ill older adults. }\end{array}$ & $\begin{array}{l}\text { Galli, Reis \& } \\
\text { Andrzejevski } \\
\text { (2016) }\end{array}$ & $\begin{array}{l}\text { English } \\
\text { Paraná }\end{array}$ & $\begin{array}{l}185 \text { elderly } \\
\text { patients } \\
\text { admitted to } \\
\text { the ICU for } \\
\text { more than } 24 \\
\text { hours }\end{array}$ & $\begin{array}{l}\text { Describe the } \\
\text { prevalence and } \\
\text { factors associated } \\
\text { with the use of MPI } \\
\text { and the occurrence of } \\
\text { adverse reactions } \\
\text { related to ADE in } \\
\text { critical elderly. }\end{array}$ & $\begin{array}{c}\text { Retrospective } \\
\text { analysis of } \\
\text { spontaneous } \\
\text { notifications } \\
\text { Period: } 1 \text { year }\end{array}$ & $\begin{array}{l}54,9 \% \\
\text { male. }\end{array}$ & 14 days & 71 years & - & $\begin{array}{l}49 \text { ADRs in } 33 \\
(17.8 \%) \\
\text { patients }\end{array}$ & $\begin{array}{l}23 \text { ADRs were } \\
\text { associated with } \\
\text { the drugs in the } \\
\text { Beers } \\
\text { Criterion. }\end{array}$ & -. \\
\hline 15 & $\begin{array}{l}\text { Adverse events in the } \\
\text { intensive care unit: } \\
\text { impact on mortality } \\
\text { and length of stay in a } \\
\text { prospective study }\end{array}$ & $\begin{array}{l}\text { Roque, Tonini } \\
\& \text { Melo (2016) }\end{array}$ & $\begin{array}{l}\text { Portuguese } \\
\text { Rio de } \\
\text { Janeiro }\end{array}$ & $\begin{array}{l}355 \text { adult } \\
\text { patients in } \\
\text { the ICU }\end{array}$ & $\begin{array}{l}\text { Assess the occurrence } \\
\text { of adverse events and } \\
\text { their impact on length } \\
\text { of stay and mortality } \\
\text { in the intensive care } \\
\text { unit (ICU). }\end{array}$ & $\begin{array}{c}\text { Prospective } \\
\text { analysis of } \\
\text { medical records. } \\
\text { IHI Trackers } \\
\text { Naranjo's } \\
\text { algorithm } \\
\text { Period: } 1 \text { year }\end{array}$ & $\begin{array}{c}189 \\
53,2 \% \\
\text { female }\end{array}$ & $\begin{array}{c}34 \text { dias } \\
\text { with } \mathrm{AE} \\
\\
15 \\
\text { without } \\
\mathrm{AE}\end{array}$ & $\begin{array}{l}60,2 \\
\text { years }\end{array}$ & $\begin{array}{c}79 \text { EAM } \\
(24,4 \%) \\
\text { em } 21 \\
(13,8 \%) \\
\text { pacientes }\end{array}$ & - & $\begin{array}{l}\text { Vasoactive and } \\
\text { sedative } \\
\text { amines }\end{array}$ & $\mathrm{N}^{\mathrm{o}}$ of medicines \\
\hline 16 & $\begin{array}{c}\text { Factors associated } \\
\text { with the occurrence of } \\
\text { adverse events } \\
\text { in critical elderly } \\
\text { patients }\end{array}$ & $\begin{array}{l}\text { Toffoletto et } \\
\text { al, (2016) }\end{array}$ & $\begin{array}{l}\text { Portuguese } \\
\text { São Paulo }\end{array}$ & $\begin{array}{l}315 \text { elderly } \\
\text { hospitalized } \\
\text { for more } \\
\text { than } 24 \\
\text { hours in the } \\
\text { ICU }\end{array}$ & $\begin{array}{l}\text { Identify the factors } \\
\text { related to the } \\
\text { occurrence of adverse } \\
\text { events in critically ill } \\
\text { elderly patients } \\
\text { admitted to the } \\
\text { intensive care unit } \\
\text { according to } \\
\text { demographic and } \\
\text { clinical } \\
\text { characteristics. }\end{array}$ & $\begin{array}{l}\text { Retrospective } \\
\text { analysis of } \\
\text { medical records } \\
\text { Moderate and } \\
\text { severe events } \\
\text { only }\end{array}$ & $\begin{array}{c}60,6 \% \\
\text { male }\end{array}$ & $\begin{array}{l}10,62 \text { dia } \\
\text { s } \\
\text { with } \\
\text { ADE } \\
\\
5,06 \\
\text { without } \\
\text { ADE }\end{array}$ & $\begin{array}{l}71,30 \\
\text { years }\end{array}$ & $\begin{array}{c}4 \text { EAM } \\
(2,5 \%) \text { do } \\
\text { total de } \\
183 \\
\text { eventos } \\
\text { adversos }\end{array}$ & - & - & - \\
\hline 17 & $\begin{array}{c}\text { Adverse Drug } \\
\text { Reactions in Patients } \\
\text { Receiving Systemic } \\
\text { Antifungal Therapy at } \\
\text { a High-Complexity } \\
\text { Hospital. }\end{array}$ & $\begin{array}{l}\text { Souza, Santos } \\
\text { \& Reis (2016) }\end{array}$ & $\begin{array}{l}\text { English } \\
\text { Minas } \\
\text { Gerais }\end{array}$ & $\begin{array}{l}183 \text { patients } \\
\text { who used } \\
\text { antifungal } \\
\text { therapy }\end{array}$ & $\begin{array}{l}\text { Determine the } \\
\text { frequency of adverse } \\
\text { drug reactions } \\
\text { associated with the } \\
\text { use of systemic } \\
\text { antifungals in patients } \\
\text { hospitalized in a } \\
\text { highly complex } \\
\text { hospital. }\end{array}$ & $\begin{array}{l}\text { Retrospective } \\
\text { analysis of } \\
\text { medical records } \\
\text { Period: } 1 \text { year }\end{array}$ & $\begin{array}{c}105 \\
(57,4 \%) \\
\text { male }\end{array}$ & - & 43 years & - & $\begin{array}{l}96 \text { ADRs in } 53 \\
\text { patients (29\%) }\end{array}$ & - & $\begin{array}{c}\text { Diagnosis of neoplasia; } \\
\text { Hospitalization time; } \\
\text { Use of } 13 \text { or more } \\
\text { medications; }\end{array}$ \\
\hline
\end{tabular}


Research, Society and Development, v. 10, n. 4, e3410413818, 2021

(CC BY 4.0) | ISSN 2525-3409 | DOI: http://dx.doi.org/10.33448/rsd-v10i4.13818

\begin{tabular}{|c|c|c|c|c|c|c|c|c|c|c|c|c|c|}
\hline 18 & $\begin{array}{l}\text { Adverse drug events } \\
\text { identified by triggers } \\
\text { at a teaching hospital } \\
\text { in Brazil. }\end{array}$ & $\begin{array}{c}\text { Giordani, } \\
\text { Rozenfeld \& } \\
\text { Martins (2014) }\end{array}$ & $\begin{array}{l}\text { English } \\
\text { Paraná }\end{array}$ & $\begin{array}{l}240 \text { patients } \\
\text { older than } 15 \\
\text { years, } \\
\text { hospitalized } \\
\text { for more } \\
\text { than } 48 \\
\text { hours. }\end{array}$ & $\begin{array}{c}\text { Evaluate the } \\
\text { incidence of ADEs } \\
\text { and to characterize } \\
\text { them in terms of } \\
\text { degree of damage, } \\
\text { medication involved } \\
\text { and patient symptoms } \\
\text { in a Brazilian } \\
\text { university hospital. }\end{array}$ & $\begin{array}{c}\text { Retrospective } \\
\text { analysis of } \\
\text { medical records } \\
\text { Period: } 7 \text { months } \\
\text { IHI Trackers } \\
\text { Causality: } \\
\text { Naranjo's } \\
\text { algorithm. }\end{array}$ & $\begin{array}{c}63,8 \% \\
\text { male }\end{array}$ & - & $\begin{array}{c}50,8 \\
\text { years }\end{array}$ & $\begin{array}{l}44 \text { EAM } \\
\text { em } 35 \\
(14,6 \%) \\
\text { pacientes }\end{array}$ & - & Anti-infectives & $\begin{array}{l}\text { Lenght of stay; } \\
\text { Use of } 10 \text { drugs or more; }\end{array}$ \\
\hline 19 & $\begin{array}{l}\text { Severe cutaneous } \\
\text { reactions to drugs in } \\
\text { the setting of a general } \\
\text { hospital. }\end{array}$ & $\begin{array}{c}\text { Grando, } \\
\text { Schmitt \& } \\
\text { Bakos (2014) }\end{array}$ & $\begin{array}{l}\text { English } \\
\text { Rio Grande } \\
\text { do Sul }\end{array}$ & $\begin{array}{c}57 \text { patients } \\
\text { with severe } \\
\text { reactions }\end{array}$ & $\begin{array}{l}\text { Evaluate the clinical } \\
\text { and epidemiological } \\
\text { aspects of serious } \\
\text { cutaneous adverse } \\
\text { drug reactions in a } \\
\text { tertiary hospital in } \\
\text { Porto Alegre, Brazil. }\end{array}$ & $\begin{array}{l}\text { Retrospective } \\
\text { analysis of } \\
\text { medical records } \\
\text { Period: } 5 \text { years }\end{array}$ & - & - & $\begin{array}{c}38,4 \\
\text { years }\end{array}$ & - & $\begin{array}{l}57 \text { patients } \\
\text { with skin } \\
\text { reactions } \\
\text { caused by } \\
\text { medication. }\end{array}$ & $\begin{array}{l}\text { Anticonvulsant } \\
\text { s } \\
\text { Antibiotics } \\
\text { Painkillers }\end{array}$ & - \\
\hline 20 & $\begin{array}{c}\text { Stevens-Johnson } \\
\text { Syndrome and Toxic } \\
\text { Epidermal Necrolysis } \\
\text { in a hospital in Distrito } \\
\text { Federal. }\end{array}$ & $\begin{array}{l}\text { Emerick, } \\
\text { Rodrigues, } \\
\text { Pedrosa, } \\
\text { Novaes \& } \\
\text { Gottems } \\
(2014)\end{array}$ & $\begin{array}{c}\text { Portugues } \\
\text { Distrito } \\
\text { Federal }\end{array}$ & $\begin{array}{l}22 \text { patients } \\
\text { hospitalized } \\
\text { with SSJ and } \\
\text { NET in the } \\
\text { ICU and } \\
\text { Burn Unit. }\end{array}$ & $\begin{array}{l}\text { Analyze the } \\
\text { demographic and } \\
\text { clinical aspects of } \\
\text { patients diagnosed } \\
\text { with Stevens Johnson } \\
\text { Syndrome (SJS) and } \\
\text { Toxic Epidermal } \\
\text { Necrolysis (NET), as } \\
\text { well as to identify } \\
\text { health professionals' } \\
\text { actions for the } \\
\text { management of } \\
\text { adverse drug } \\
\text { reactions (ADR) in a } \\
\text { public hospital in the } \\
\text { District. Federal, } \\
\text { Brazil. }\end{array}$ & $\begin{array}{l}\text { Retrospective } \\
\text { analysis of } \\
\text { medical records } \\
\text { Period: } 7 \text { years }\end{array}$ & Female & - & - & - & $\begin{array}{l}22 \text { patients } \\
\text { with SSJ and } \\
\text { NET due to } \\
\text { medications. }\end{array}$ & Antiepileptics. & - \\
\hline
\end{tabular}

Source: Research Protocol (2019). 


\section{Discussion}

In this review $50 \%$ (10) of the studies came from the southeast region, from these, 20\% (4) concentrated in SP. These data corroborate the reports of The Royal Society (2011) which verified spatial heterogeneity in scientific production, with a large concentration of studies in the southeast region, and listed São Paulo as the region responsible for about $20 \%$ of scientific production in Brazil.

In the methodology adopted, there was a prevalence $70 \%$ (14)of studies with retrospective review of data. According to some authors in the identification of ADEs, retrospective review of medical records is one of the most widely used methods, as well as spontaneous reporting, but they point out the retrospective methodology usually has limitations in identifying associated risk factors and determining the causality chain ${ }^{[38,39.40]}$. In addition, Franklin's study at a surgical center in 2010 found the lack of complete data in patient records was a factor that hinder the ADEs identification, from the 207 records analyzed, 25\% (51/207) did not have complete data and 17\% (35) did not have accessible laboratory data (Franklin, Birch, Sachter \& Barber, 2010).

In literature, there are variations in the identification rates of $\mathrm{AE}$ found through trackers (IHI or other triggers), some studies show rates of only $2 \%$, while in others the identification rates are approximately $24 \%$ (Classen, Pestotnik, Evans, Burke \& Battles, 1991; Davies, Green, Mottram \& Pirmohamed, 2006). In addition to the lack of information in medical records, other factors that may interfere are the number of trackers used to identify events and the rate of change that trackers undergo when adapted to the reality of each health service (Seddon et al, 2013). There is a correlation between the event occurrence and variables such as length of stay, gender, age and presence of comorbidities. Thus, the characteristics of the studied population also directly influence the number of adverse events found (Seddon et al, 2013; Robb, Loe, Maharaj, Hamblin \& Seddon, 2017; Varallo et al, 2017).

This information corroborates the data found in the selected articles, where studies using the IHI triggers identified 120 (Roque et al., 2018) and 44 (Giordani,et al., 2014) ADEs, and 79 (Roque et al., 2016) ADRs. While other trackers proposed by the authors identified 115 (Fabretti et al., 2018), 62 (Figueiredo et al., 2017) ADEs.

There is great variability in the occurrence rates of ADES and/or ADRs within hospital services, this may be related to the fact that there is no gold standard to calculate the rate of AE, as well as the safety measures already implemented by health services where the studies were performed (Griffin \& Resar, 2009).

From the 16 articles, which characterized the patients'gender, 11 (69\%), had a prevalence of male hospitalization. This data is consistent with some studies in which men present characteristics that potentiate the disease, due to greater neglect of health and low use of primary and secondary care, being at greater risk of clinical decompensation (Camarano, 2002; Almeida, Mafra, Silva \& Kanso, 2015).

Fifty per cent (7) of the 14 articles which identify the patients age had a mean age greater than 60 years. Among the factors that predispose the patient to ADEs, the following stand out: extremes of age, severity of the clinical condition, comorbidities and polytherapy, which would characterize geriatric patients as a risk group. The geriatric population is possibly the most medicalized group in society due to the high incidence of chronic degenerative diseases among them, and the progressive decrease in functional capacity and self-medication. In addition, during the aging process there are physiological changes interfering in pharmacokinetic (drug absorption, metabolism and excretion) and pharmacodynamic processes, which predispose this group to toxicity-related interactions, drug interactions, and adverse reactions (Silva, Ribeiro, Klein \& Acurcio, 2012; Carvalho et al., 2012).

Regarding the drugs that caused ADEs, we highlight the antimicrobial and central nervous system drugs. In the study by Almeida, Castro and Caldas (2011) anti-infectious drugs were also the main drugs related to ADEs. These drug classes were 
related to severe skin reactions such as Steven-Johnson Syndrome and Toxic Epidermal Necrolysis (Grando et al., 2014; Emerick et al., 2014).

The risk factors associated with the occurrence of myocardial infarction were the length of stay, polytherapies and previous history of myocardial infarction. Regarding length of stay, two studies showed differences of 5 (Toffoletto et al., 2016) and 15 (Roque et al., 2016) days, respectively, when comparing patients with and without ADE. These findings corroborate the results found by Briant, Ali, Lay-yee, and Davis (2004) and Rozenfeld, Giordani and Coelho (2013), where the first author identified patients that were affected by drug-related damage had their length of stay increased by 6 days, and the second author showed the time difference between the two groups. These variables seem to be interdependent, since as events increase length of stay, prolonged hospitalization is closely related to the increased risk of suffering care events and increased cost to health services.

Obviously, there are several methods for searching and identifying adverse events, despite the active search, such as the use of trackers. This kind of study had identified the largest number of adverse winds, and spontaneous reporting was biased to underreport events, it should not be replaced by another active search methodology. For while active search usually mobilizes only a few authors involved in event screening, spontaneous reporting spreads the culture of harm reduction and promotion of patient safety among all participants in the care process (Sari et al., 2007). Thus, health services should structure both forms of event identification (Rutberg et al., 2014).

The drug-related events are the third leading cause of adverse events within health services usually due to medication errors. In the structure of services the pharmacist represents one of the best professionals to identify, correct or reduce potential risks associated with therapy (Pepe \& Osório-de-Castro, 2000), but in this review only 2 studies used the pharmacist to identify ADEs. Due to his vast knowledge of medicines and therapeutics, the pharmacist is trained to act directly in the identification, detection, prevention and management of medication errors, drug interactions, and adverse reactions within the multiprofessional team (Sriram et al., 2011). There are even studies that have already shown a reduction in the number of medication errors in institutions where pharmacists performed interventions with the clinical staff (Planas, 2004). In this sense, the pharmaceutical intervention, by reducing the number of adverse events, should be used to increase the quality of care and decreases hospital costs.

\section{Conclusion}

In Brazil, there is variability in the occurrence of ADEs in hospitalized patients, and because these events are associated with increased length of stay, patient damage, and increased hospital costs, health services should implement strategies for their identification. Performing analysis of each event individually in order to identify possible weaknesses in the processes within hospitals and propose corrective measures that promote patient safety are urgent. Therefore, it is essential that new studies are carried out focused on the identification and risk factors, but mainly prevention factors.

\section{References}

Agência Nacional de Vigilância Sanitária (Brasil) (2013). ANVISA. Segurança do paciente e qualidade em serviços de saúde. Boletins Informativo.

Almeida, A. V., Mafra, S. T. C., Silva, E. P., \& Kanso, S. (2015). The feminization of old age: a focus on the socioeconomic, personal and family characteristics of the elderly and the social risk, Textos\&Contextos; 14(1): 115-31

Almeida, M. R., Castro, L. L. C. \& Caldas, E.D. (2011). Conhecimentos, práticas e percepção de risco do uso de medicamentos no Distrito Federal. RevCiêncFarm Básica Apl; 32(1):225-232.

Andrade, P. H. S., Lobo, I. M. F., \& Silva, W. B. (2017). Risk factors for adverse drug reactions in pediatric inpatients: A cohort study. PLoSOne; 12(8): e0182327. 
Basile, L. C., Santos, A., Stelzer, L. B., Alves, R. C., Fontes, C. M. B., Borgato, M. H., Papini, S. J., \& Lima, S. A. M. (2019). Análise das ocorrências de incidentes relacionados aos medicamentos potencialmente perigosos dispensados em hospital de ensino. Rev Gaúcha Enferm; 40.

Beccaria, L. M., Pereira, R. A. M., Contrin, L. M., Lobo, S. M. A., \& Trajano, D. H. L. (2009). Nursing care adverse events at an intensive care unit. RevBras Ter Intensiva; 21:276-82.

Briant, R., Ali, W., Lay-Yee, R. \& Davis, Peter. (2004). Representative case series from public hospital admissions 1998: drug and related therapeutic adverse events. NZMed J;117(1188):U747.

Camarano, A. A. (2002). Envelhecimento da população brasileira: uma contribuição demográfica. In: Freitas EV et al, organizadores. Tratado de Geriatria e Gerontologia.:Guanabara Koogan.

Capucho, H. C., Arnas, E. R., \& Cassiani, S. H. B. (2013). Patient safety: a comparison between handwritten and computerized voluntary incident reporting. RevGauchaEnferm; 34(1):164-172.

Carvalho, M. F. C., Romano-Lieber, N. S., Bergsten-Mendes, G., Secoli, S. R., Ribeiro, E., Lebrao, M. L., \& Duarte, Y. A. O. (2012). Polifarmácia entre idosos do Município de São Paulo - Estudo SABE. RevBrasEpidemiol; 15(4): 817-27.

Chaves, E. C. (2017). Epidemiological, clinical and evolutionary aspects of tuberculosis among elderly patients of a university hospital in Belém, Pará. Revbras geriatr gerontol; 20(1): 45-55.

Classen, D. C., Pestotnik, S., Evans, R., Burke, J., \& Battles., J. (1991). Computorized surveillance of adverse drug events in hospital patients. JAMA; 266(20):2847-51.

Classen, D. C., Pestotnik, S., Evans, R.., Lloyd, J. F., \& Burke, J. P. (1997). Adverse drug events in hospitalized patientes. Excess length of stay, extra costs, and atributable mortality. JAMA; 277(4): 301-306.

Classen, D. C., Resar, R., Griffin, F., Federico, F., Frankel, T., Kimmel, N., Whittington, J. C., Frankel, A., Seger., A., \& James, B. C. (2011). Global trigger tool' shows that adverse events in hospitals may be ten times greater than previously measured. Health Aff; 30(4):581-9.

Davies, E. C., Green, C. F., Mottram, D. R., \& Pirmohamed, M. (2006). Adverse drug reactions in hospital in-patients: a pilot study. J Clin Pharm Ther; 31(4):335-41

Dolores-Menendez, M., Rancano, I., Garcia, V., Vallina, C., Herranz, V., \& Vazquez, F. (2010). Use of different patient safety reporting systems: much ado about nothing? Rev Calid Asist; 25(4): 232-236.

Emerick, M. F. B., Rodrigues, M. M. T., Pedro, D. M. A. S., Novaes, M. R. C. G., \& Gottems, L. B. D. (2014). Síndrome de Stevens-Johnson e necrólise epidérmica tóxica em um hospital no Distrito Federal. RevBrasEnferm; 67 (6): 898-904.

Fabretti, S. C., Brassica, S. C., Cianciarullo, M. A., \& Romano-Lieber, N. S. (2018). Rastreadores para a busca ativa de eventos adversos a medicamentos em recém-nascidos. CadSaude Publica; 34 (9): e00069817.

Figueiredo, T. P., Groia, R. C. S., Barroso, S. C. C., Nascimento, M. M. G., \& Reis, A. M M. (2017). Factors associated with adverse drug reactions in older inpatients in teaching hospital. Int J ClinPharm; 39(4): 679-685.

Forster, A. J., Worthington, J. R., Hawken, S., Bourke, M., Rubens, F., Shojania, K., \& Carl van Walraven. (2011). Using prospective clinical surveillance to identify adverse events in hospital. BMJ Qual Saf; 20:756-63.

Franklin, B. D., Birch, S., Schachter, M., \& Barber, N. (2010). Testing a trigger tool as a method of detecting harm from medication errors in a UK hospital: a pilot study. Int J Pharm Pract; 18(5):305-11.

Gadelha, G. O., Paixão, H. C. S., Prado, P. R., Viana, R. A. P. P., \& Amaral, T. L. M. (2018). Risk factors for death in patients with non-infectious adverse events. RevLatAm Enfermagem; 26: e3001.

Galli, T. B., Reis, W. C., \& Andrzejevski, V. M. (2016). Potentially inappropriate prescribing and the risk of adverse drug reactions in critically ill older adults. PharmPract; 14(4): 818 .

Giordani, F., Rozenfeld, S., \& Martins, M. (2014). Adverse drug events identified by triggers at a teaching hospital in Brazil. BMC PharmacolToxicol; 15: 71.

Grando, L. R., Schmitt, T. A. B., \& Bakos, R. M. (2014). Severe cutaneous reactions to drugs in the setting of a general hospital. AnBrasDermatol; 89 (5): 758-62.

Griffin, F. A., \& Resar, R. K. (2009). IHI Global Trigger Tool for Measuring Adverse Events (Second Edition). Innovation Series white paper. Cambridge, Massachusetts: Institute for Healthcare Improvement.

Haukland, E. C., Plessen, C., Nieder, C., \& Vonen, B. (2017). Adverse events in hospitalised cancer patients: a comparison to a general hospital population. Acta Oncol; 56(9): 1218-1223.

Institute of Medicine (IOM) (1999). Committe on Quality of Health Care in Americna; To err is human: Building a safer health system. NationalAcademy Press.

Kimelblatt, B. J., Young, S. H., Heywood, P. M., Mandala, A. R., Gendelman, S., \& Mehl, B. (1988). Improved reporting of adverse drug reactions. Am J hosp Pharm; 45 (5):1086-1089.

Nascimento, C. C. P., Toffoletto, M. C., Gonçalves, L. A., Freitas, W. G., \& Padilha, K. G. (2008). Indicators of healthcare results: analysis of adverse events during hospital stays. Rev LatinoamEnferm; 16:746-51. 
Nunes, A. M. C. (2000). Conceitos básicos de Farmacovigilância. In: CASTRO, C.G.S.O. (Coord.). Estudos de utilização de medicamentos: noções básicas. Rio de Janeiro: Editora Fiocruz; 106-126p.

Martins, A. C., Giordani, F., Guaraldo, L., Tognoni, G., \& Rozenfeld, S. (2018). Eventos adversos a medicamentos identificados em pacientes hospitalizados no Brasil pelas listagens de códigos da Classificação Internacional de Doenças (CID-10). CadSaude Publica; 34 (12): e00222417.

Mendes, W., Martins, M., Rozenfeld, S., \& Travassos, C. (2009). The assessment of adverse events in hospitals in Brazil. Int J Qual Health Care; 21(4):27984.

Ministério da Saúde. Agência Nacional de Vigilância Sanitária. Relatório de notificações 2006-2013 [Internet]. Sistema Nacional de Notificações para a Vigilância Sanitária: módulo de notificação. http://www.anvisa.gov.br/hotsite/notivisa/manual/ea_medicamento_profissional.pdf

Nunes, B. M., Xavier, T. C., \& Martins, R. R. (2017). Problemas relacionados a medicamentos antimicrobianos em unidade de terapia intensiva neonatal. Revbras ter intensiva; 29(3):331-336.

Pepe, V. L. E., \& Osório-de-Castro, C. G. S. (2000). A interação entre prescritores, dispensadores e pacientes: informação compartilhada como possível benefício terapêutico. Cad Saúde Pública; 16(3): 815-822.

Planas, M. C. G. (2004). (Cord.). Libro de la Sociedad Española de Farmacia Hospitalaria (3th.ed.) Madrid.

Ramos, G. V., Japiassu, A. M., Bozza, F. A., \& Guaraldo, L. (2018). Preventable adverse drug events in critically ill HIV patients: Is the detection of potential drug-drug interactions a useful tool? Clinics; 73: e148.

Ribeiro, M. R., Motta, A. A., Marcondes-Fonseca, L. A., Kalil-Filho, J., \& Giavina-Bianchi, P. (2018). Increase of 10\% in the Rate of Adverse Drug Reactions for Each Drug Administered in Hospitalized Patients. Clinics; 73: e185.

Robb, G., Loe, E., Maharaj, A., Hamblin, R., \& Mary \& Sedon. (2017). Medication-related patient harm in New Zealand hospitals. N Z Med J; 130(1460): 2132 .

Roque, K. E., Silva, A. R. G., Santos, M. H. B. A., \& Melo, E. C. P. (2018). Fatores de risco associados à hipoglicemia e análise de eventos adversos em uma terapia intensiva. Texto contexto - enferm, 27(3): e3350016.

Roque, K. E., Tonini, T., \& Melo, E. C. P. (2016). Adverse events in the intensive care unit: impact on mortality and length of stay in a prospective study. Cad Saúde Pública; 32(10): 1678-4464.

Rozenfeld, S., Giordani, F., \& Coelho, S. (2013). Eventos adversos a medicamentos em hospital terciário: estudo piloto com rastreadores. Rev Saúde Pública; 47(6): 1102-1111.

Rutberg, H., Risberg, M. B., Sjodahl, R., Nordqvist, P., Valter, L., \& Nilsson, L. (2014). Characterisations of adverse events detected in a university hospital: a 4-year study using the Global Trigger Tool method. BMJ Open; 4(5): e004879.

Santos, G. A. S., \& Boing, A. C. (2018). Mortalidade e internações hospitalares por intoxicações e reações adversas a medicamentos no Brasil: análise de 2000 a 2014. Cad Saúde Pública; 34(6): e00100917.

Sari, A. B., Sheldon, T. A., Cracknell, A., \& Turnbull, A. (2007). Sensitivity of routine system for reporting patient safety incidents in an NHS hospital: retrospective patient case note review. BMJ; 334:79.

Seddon, M. E., Jackson, A., Cameron, C., Young, M. L., Escott, L., Maharaj, A., \& Miller, N. (2013). The Adverse Drug Event Collaborative: a joint venture to measure medication-related patient harm. N Z Med J; 126(1368):9-20.

Silva, A. L., Ribeiro, A. Q., Klein, C. H., \& Acurcio, F. A. (2012). Utilização de medicamentos por idosos brasileiros, de acordo com a faixa etária: um inquérito posta. Cad Saúde Pública; 28(6):1033-1045.

Souza, M. C. P., Santos, A. G., \& Reis, A. M. M. (2016). Adverse Drug Reactions in Patients Receiving Systemic Antifungal Therapy at a High-Complexity Hospital. J Clin Pharmacol; 56 (12): 1507-1515.

Sriram, S., Ghasemi, A., Ramasamy, R., Devi, M., Balasubramanian, R., Ravi, T. K., \& Sabzhabaee, A. M. (2011). Prevalence of adverse drug reactions at a private tertiary care hospital in south India. J Res MedSci; 16(1):16-25.

The Royal Society. (2011). Knowledge, networks and nations: Global scientific collaboration in the 21st century. London: The Royal Society. <https://royalsociety.org/policy/projects/ knowledge-networks-nations/report/>.

Toffoletto, M. C., Barbosa, R. L., Andolhe, R., Oliveira, E. M., Ducci, A. J., \& Padilha, K. G. (2016). Fatores relacionados à ocorrência de eventos adversos em pacientes idosos críticos. RevBrasEnferm; 69(6):1039-1045.

Varallo, F. R., Dagli-Hernandez, C., Pagotto, C., Nadai, T. R., Herdeiro, M. T., \& Mastroianni, P. C. (2017). Confounding Variables and the Performance of Triggers in Detecting Unreported Adverse Drug Reactions. ClinTher; 39(4).

Veloso, R. C. S. G., Figueredo, T. P., Barroso, S. C. C., Nascimento, M. C. G., \& Reis, A. M. M. (2019). Factors associated with drug interactions in elderly hospitalized in high complexity hospital. CienSaude Colet; 24(1): 17-26.

Venulet, J., \& Ham, M. (1996). Methods for monitoring and documenting adverse drug reactions. Int J ClinPharmacolTher; 34(3): 112-129.

Vicent, C. (2010). Segurança do paciente: orientações para evitar os eventos adversos. Yendis. 4p. 
Research, Society and Development, v. 10, n. 4, e3410413818, 2021

(CC BY 4.0) | ISSN 2525-3409 | DOI: http://dx.doi.org/10.33448/rsd-v10i4.13818

World Health Organization (WHO). (2002). Safety monitoring of medicinal products. The importance of pharmacovigilance. Organización Mundial de la Salud.

World Health Organization (WHO). (2009). The Conceptual Framework for the International Classification for Patient Safety. World Alliance for Patient Safety. 